\title{
Management of Esophageal Achalasia in Quebec
}

\author{
Catherine Pouyez ${ }^{\mathrm{a}}$,, Elissaveta Neshkova ${ }^{\mathrm{a}}$, Daniel von Renteln ${ }^{\mathrm{a}}$, \\ Mickael Bouin ${ }^{\mathrm{a}}$
}

\begin{abstract}
Background: Esophageal achalasia is a defective relaxation of the lower esophageal sphincter with a loss of esophageal peristalsis causing dysphagia. Treatment can be Heller myotomy, pneumatic balloon dilation, Botox injections, peroral endoscopic myotomy (POEM) or medical. The main objective of the study was to measure the extent of post-treatment dysphagia depending on the type of treatment.
\end{abstract}

Methods: This was a retrospective study conducted at the Centre Hospitalier de l'Universite de Montreal (CHUM) between 2011 and 2017. All patients with manometric diagnostic of achalasia in our department were included. Data were collected with the electronic health record and a standardized post-treatment telephone survey to evaluate the extent of dysphagia and the use of proton pump inhibitor (PPI).

Results: A total of 169 patients were included. The most frequent treatments were Heller myotomy (60\%), Botox injection (18\%) and endoscopic balloon dilation (16\%). There was a significant difference in the management of patients treated at the CHUM and outside the CHUM for the frequency of pneumatic dilation (28 vs. $7 \%$; $\mathrm{P}=$ 0.001 ) and Heller myotomy (49 vs. $69 \% ; \mathrm{P}=0.02$ ). An Eckardt score $\leq 3$ was found in $80 \%$ of patients. No significant score difference was found between the CHUM and outside CHUM groups or between the different treatments. The post-treatment use of PPI was of $49 \%$ and was not significantly different depending on the site or the type of treatment.

Conclusion: Heller myotomy stays the most frequent treatment, especially outside our tertiary center. The rate of post-treatment dysphagia is low no matter the treatment choice. The use of PPI stays frequent regardless of the management site or the type of treatment used.

Keywords: Esophageal achalasia; Management in Quebec; Eckardt score

Manuscript submitted August 22, 2019, accepted September 12, 2019

aCentre Hospitalier de l'Universite de Montreal (CHUM), 1051 Sanguinet St., Montreal, QC, Canada

${ }^{b}$ Corresponding Author: Catherine Pouyez, Centre Hospitalier de l'Universite de Montreal (CHUM), 1051 Sanguinet St., Montreal, QC, H2X 3E4, Canada. Email: catherine.pouyez@umontreal.ca

doi: https://doi.org/10.14740/jocmr3963

\section{Introduction}

Primary esophageal achalasia is caused by an inflammatory and degenerative process of myenteric plexus neurons, causing a deficit of lower esophageal sphincter (LES) relaxation during swallowing and loss of peristalsis in the esophagus. The cause of this degeneration is unknown [1]. The annual incidence is about 1 per 100,000 and affects men and women of all ages, most often between the ages of 30 and 60 [2]. The main symptoms are dysphagia with solids and liquids, regurgitation of undigested food or saliva, chest pain and weight loss [3]. The diagnosis is made by esophageal manometry, which shows the lack of LES relaxation and loss of esophageal peristalsis [4]. Secondary achalasia is caused by several diseases such as amyloidosis, sarcoidosis, Chagas disease and several neoplasms, causing an esophageal motor disorder like primary achalasia [5]. The treatment then depends on the cause of the secondary achalasia.

There are several types of treatment that aim at reducing the tone of the LES. Surgical treatment with Heller myotomy is the treatment of choice because of its effectiveness and durability [6]. Botulinum toxin injection is reserved for patients who cannot undergo invasive treatment; it is less effective in the long term than other treatments as more than half of the patients require a second injection after 6 - 12 months [7]. Endoscopic balloon dilatation is more effective and more durable than Botox [6]. It can be offered as an alternative to surgery. According to some studies, it is not inferior to Heller myotomy in the short term [8], but loses its effectiveness after 5 - 10 years [9]. Peroral endoscopic myotomy (POEM) is the newest treatment for achalasia and is less invasive than Heller myotomy. Current data do not show any difference between this endoscopic method and Heller myotomy with respect to complications and short-term success rate $[10,11]$. Medical treatment with a nitrate derivative or a calcium channel blocker is generally reserved for patients with few symptoms or nonsurgical candidates, as this is the least effective treatment. With any given treatment, up to $20 \%$ of patients will require a second treatment after 5 years [3].

The efficacy of treatment is assessed using the Eckardt score. It evaluates the four main symptoms of achalasia: dysphagia, regurgitation, retrosternal pain and weight loss, graded from 0 to 3 . An Eckardt score of $\leq 3$ is considered a clinical success [5].

The Centre Hospitalier de l'Universite de Montreal (CHUM) is a tertiary center in the Montreal area. A large part of the manometries of the region are carried out in our center, but treatment is done in the hospitals that referred the patients. Prior to this study, we did not have data on the treatments and 


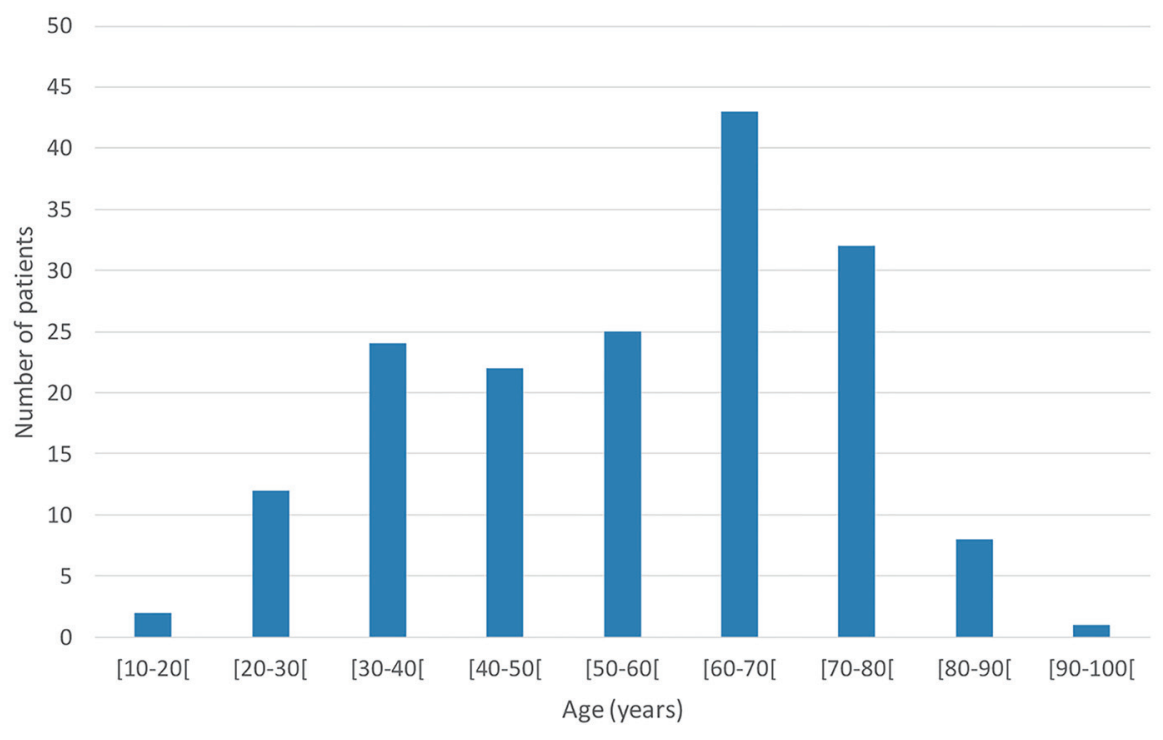

Figure 1. Age distribution of patients with esophageal achalasia.

their success rate after patients are diagnosed at the CHUM. The main objective of this study was to determine the importance of residual dysphagia after treatment according to the type of treatment received and the place of care. The secondary objectives were to determine the type of treatment received at the CHUM compared to other hospitals in the region, to evaluate the need for a second treatment and to quantify post-treatment proton pump inhibitor (PPI) intake.

\section{Materials and Methods}

This was a retrospective study from January 2011 to December 2017. All patients diagnosed with esophageal achalasia in the CHUM neurogastroenterology and digestive motility unit were included.

The study was approved by the Ethics Committee of the CHUM Research Center and the Institutional Review Board.

All patients diagnosed with achalasia as indicated on the manometry report were included in the study and their electronic record was reviewed. Age, gender and manometric data were obtained using electronic records. All patients were contacted by telephone in two stages, in 2013 for the manometry from 2011 to 2012 and in 2018 for the manometry from 2013 to 2017. All patients were asked the same standardized questionnaire to collect information on the treatments received (type, number, place and year of treatment). The post-treatment Eckardt score at the time of the telephone questionnaire was obtained. The intake of PPI was also questioned. The delay between the onset of symptoms and the manometric diagnosis was obtained only for patients who were questioned less than 1 year after diagnostic manometry, thus minimizing memory bias.

The collected data were analyzed with the XLSTAT software for Microsoft Excel. The average Eckardt score at the CHUM was compared to the outside-CHUM score by a $t$-test. The analysis was also repeated for the different treatments by comparing the data collected 1 - 2 years vs. 3 - 5 years after treatment. An analysis of variance (ANOVA) was done to compare the post-treatment Eckardt score depending on the type of treatment. The types of treatments performed at CHUM vs. outside-CHUM were compared using a Chi-square test. This test was also used to compare PPI intake and for the need of a second treatment depending on location and type of treatment. The significance level used was $5 \%$. When the Chi-square result was statistically significant, we performed post-hoc pairwise comparisons with a Bonferroni correction.

\section{Results}

A total of 285 patients were screened in the study. Of the patients, $34 \%$ were not reachable by phone (change of address or death), and $6 \%$ of patients were excluded because they refused to participate or could not communicate by telephone (deafness, language barrier or cognitive impairment). Sixty percent of patients $(n=169)$ were included in the study.

\section{Patients' characteristics}

The mean age of the patients was $56 \pm 17$ years with $53 \%$ of men (Fig. 1). The age and sex of patients depending on the treatment received are shown in Table 1. The unreachable group was not different in terms of age and sex ( $59 \pm 21$ years with 54\% men, NS). The average time between onset of symptoms and diagnosis was $24 \pm 24$ months. The most commonly reported symptom was dysphagia $(67 \%)$, followed by blocking sensation (23\%), weight loss $(23 \%)$ and reflux $(16 \%)$.

Twenty-two percent $(n=38)$ did not receive treatment for a variety of reasons: patient refusal, mild symptoms, or waiting for treatment. Of the treated patients, $47 \%$ (61) were treated at the CHUM. 
Table 1. Characteristics of Patients Depending on the Type of Treatment Received

\begin{tabular}{llllll}
\hline & Heller myotomy & Botox injection & Dilation & POEM & Medical treatment \\
\hline Patients, $\mathrm{n}(\%)$ & $79(60)$ & $24(18)$ & $22(16)$ & $4(3)$ & $3(2)$ \\
Mean age, years & 51 & 68 & 54 & 56 & 62 \\
Male sex, n (\%) & $37(47)$ & $11(46)$ & $16(73)$ & $3(75)$ & $1(33)$ \\
Female sex, $\mathrm{n}(\%)$ & $42(53)$ & $13(54)$ & $6(27)$ & $1(25)$ & $2(67)$ \\
\hline
\end{tabular}

POEM: peroral endoscopic myotomy.

\section{Treatments received}

Overall, $60 \%$ had a Heller myotomy, $18 \%$ Botox injection, $16 \%$ dilation, $3 \%$ POEM and $2 \%$ medical treatment. The type of treatment was decided by the treating gastroenterologist with the patient's consent [5].

\section{Eckardt score}

The average post-treatment Eckardt score was 2.24, all treatments combined. Overall, $80 \%$ of patients achieved an Eckardt score $\leq 3$ (Fig. 2). Eckardt scores were not significantly different regardless of the type of treatment received (Fig. 3). Eckardt scores obtained $1-2$ years after treatment were compared to those obtained 3 - 5 years after treatment. No significant difference was observed for the Eckardt score between these post-treatment delays (Fig. 4).

\section{Post-treatment PPI intake}

Post-treatment PPI intake was $49 \%$, all treatments combined. The percentage of patients taking PPIs was not significantly different regardless of the treatment received (Fig. 5). PPI intake was greater 3 - 5 years after treatment than $1-2$ years (36\% vs. $67 \%, \mathrm{P}<0.001)$ (Fig. 6).

\section{Second treatment}

The need for a second treatment was $24 \%$ for all treatments combined. It was significantly lower in the Heller myotomy group (Heller myotomy versus dilation, $6 \%$ vs. $76 \%$, $\mathrm{P}<0.001$; Heller myotomy versus Botox, $6 \%$ vs. $50 \%, \mathrm{P}<0.001$ ) (Fig. 7).

\section{Treatment at CHUM vs. outside CHUM}

The choice of treatments was significantly different between the group of patients treated at the CHUM or outside the CHUM. Heller myotomy was significantly less frequent in the CHUM than outside CHUM ( 49 vs. $69 \%, \mathrm{P}=0.001$ ), whereas endoscopic dilatation was significantly more frequent in the CHUM than outside CHUM (28 vs. $7 \%, \mathrm{P}=0.02$ ). The frequency of Botox injections, medical treatment and POEM was not significantly different between CHUM and outside the CHUM (Fig. 8).

There was no significant difference for the Eckardt scores

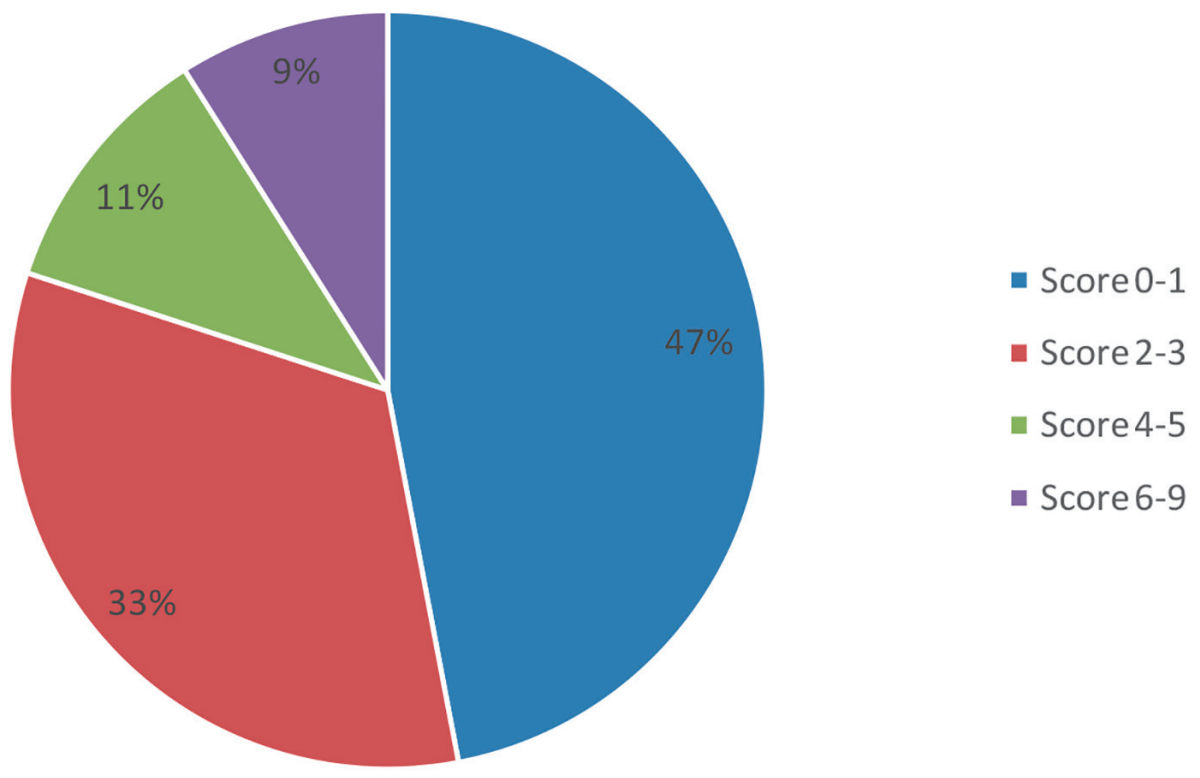

Figure 2. Eckardt score distribution after treatment of esophageal achalasia. 


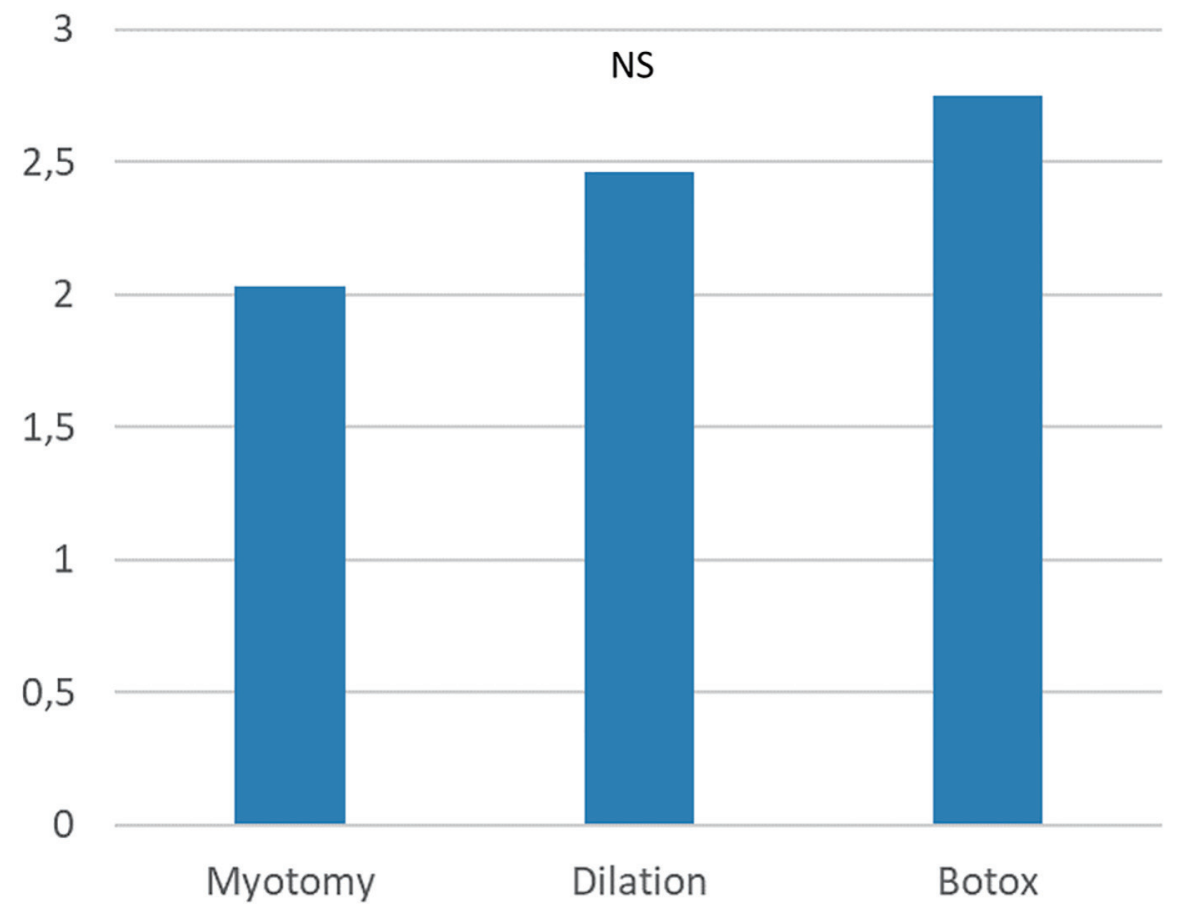

Figure 3. Comparison of post-treatment Eckardt score depending on the type of treatment for esophageal achalasia.

(Fig. 9) nor the post-treatment PPI intake (Fig. 10) between the groups treated at the CHUM and outside the CHUM.

\section{Discussion}

This study examined the treatments results for esophageal achalasia in the Montreal area of Quebec. It concerned a long period of observation ( 7 years) and allows, for the first time in Quebec, to objectify differences in care between a tertiary center and secondary or community centers.

Our study demonstrated that post-treatment symptomatology was equivalent regardless of the site of care or the type of treatment (Heller myotomy, balloon dilatation or Botox injection). Wang's meta-analysis showed a higher initial remission rate for dilatation vs. Botox and for Heller myotomy vs. dilation [5]. In contrast, several other studies did not demonstrate a difference between dilatation and Heller myotomy, which concurs

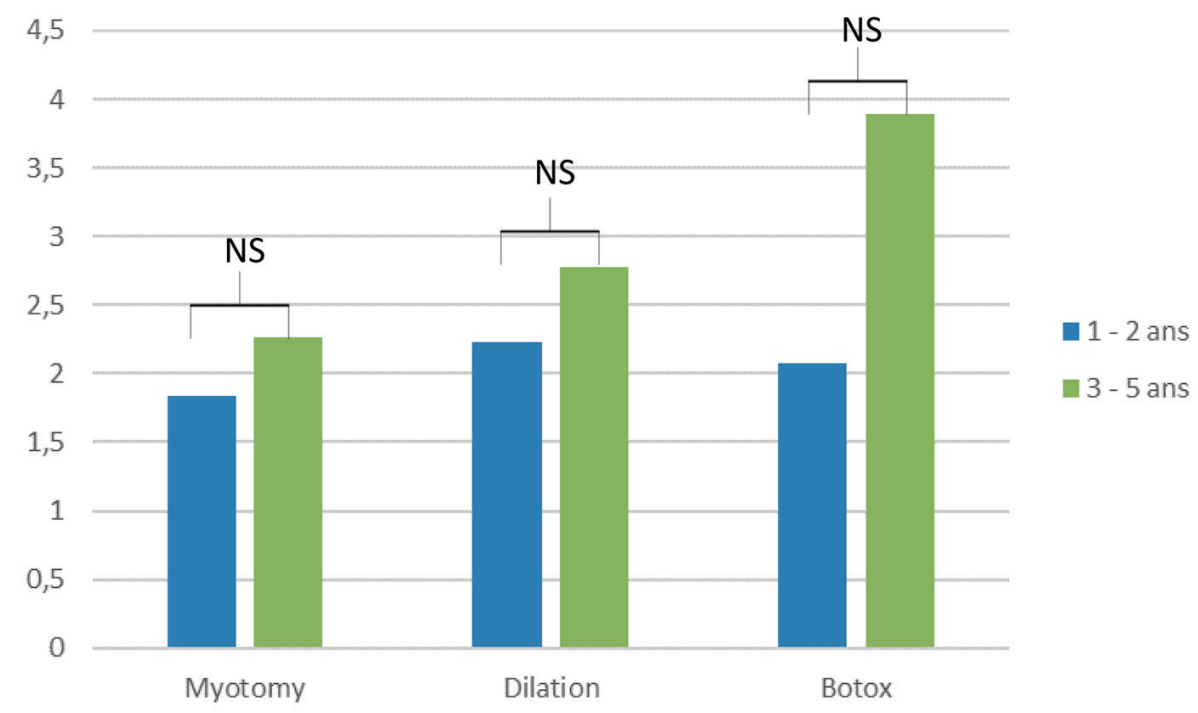

Figure 4. Comparison of post-treatment Eckardt score depending on the delay after the treatment for esophageal achalasia. 


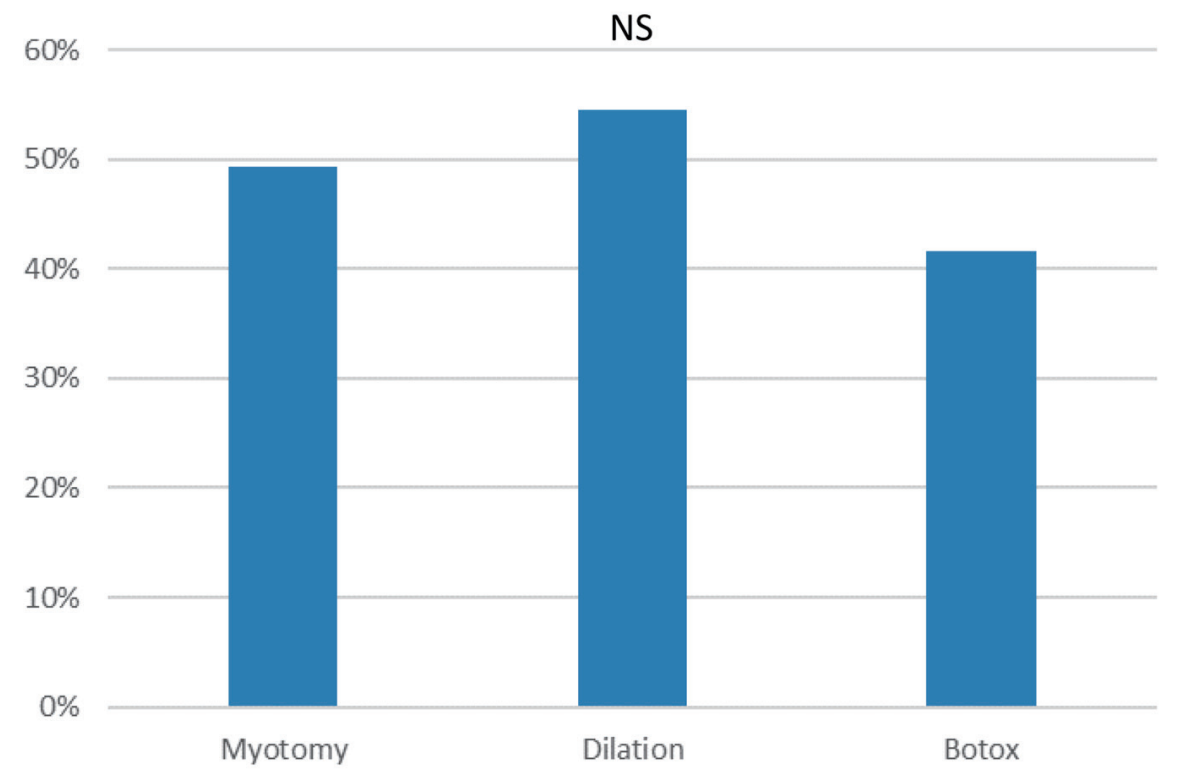

Figure 5. Percentage of patients taking proton pump inhibitor depending on the type of treatment for esophageal achalasia.

with the results of our study $[4,12,13]$. No significant difference was found between Eckardt scores 1 - 2 years versus 3 - 5 years after treatment, but there was a trend towards increasing the Eckardt score for Botox after 3 - 5 years. This trend is probably due to the decline in Botox efficacy with time, which is demonstrated in several studies. The remission rate decreases from $70 \%$ at 3 months to $41 \%$ at 12 months according to Stavropoulos et al [2], and from $48 \%$ at 1 year to $9 \%$ at 5 years according to Jung et al [7]. Several studies also show an efficacy loss for dilation after 5 - 6 years [1-3, 7, 14], which has not been demonstrated in our study. This could be explained by the fact that there is less than 5 years of follow-up for most patients.

In our study, the need for a second treatment was higher following a dilation or injection of Botox than post-Heller myotomy. These results are consistent with the literature with more than $50 \%$ recurrence of symptoms at 1 year after Botox injection according to Patel et al [1]. A meta-analysis also demonstrated that more than one-third of dilated patients had a relapse requiring a second treatment. It is possible that these additional treatments lowered the Eckardt scores 3 - 5 years af-

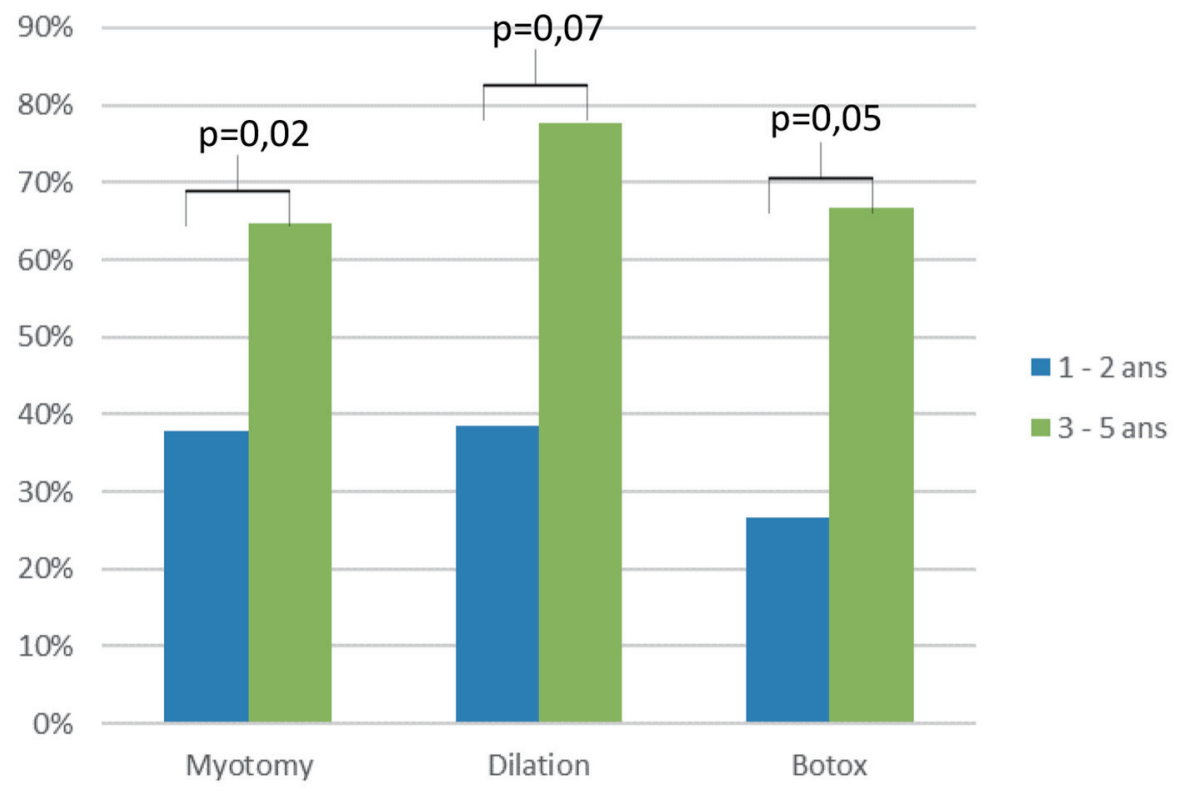

Figure 6. Percentage of patients taking proton pump inhibitor depending on the delay after the treatment for esophageal achalasia. 
$100 \%$

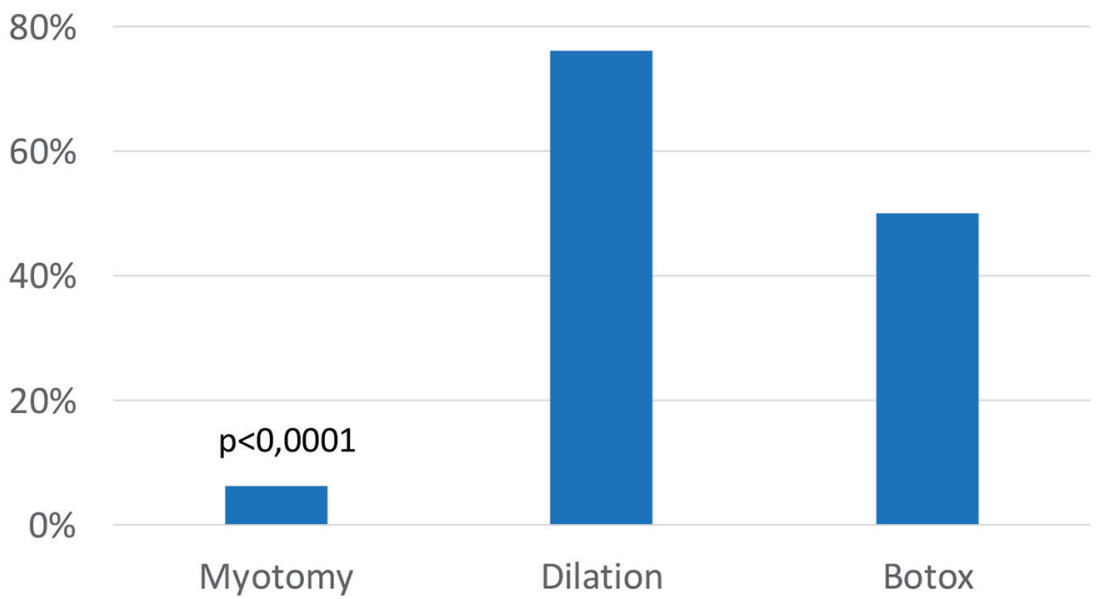

Figure 7. Percentage of patients needing a second treatment depending on the type of treatment for esophageal achalasia.

ter dilatation and Botox, suggesting that these treatments were as effective as Heller myotomy.

In our study, nearly half of patients took post-treatment PPI, regardless of the type of treatment received or the site of management. This proportion may seem quite high compared to post-treatment gastroesophageal reflux (GERD) rates reported in the literature. GERD rates of $15-35 \%$ are reported post-dilatation and 10-40\% post-Heller myotomy [2, 4, 12]. Novais et al have demonstrated the presence of GERD posttreatment with the help of pH-metry. They demonstrated a higher rate of GERD after dilatation than after Heller myotomy $(31 \%$ vs. $5 \%, P=0.0001)$. However, the epigastric burning sensation was independent of the result of $\mathrm{pH}$-metrics. Of the patients included in the study, $44 \%$ had a burning sensation and $40 \%$ of those with negative $\mathrm{pH}$-metrics still had a burning sensation [5]. In our study population, PPIs are likely to be prescribed because of patients' symptoms rather than an objec- tive test for GERD (pH-metrics), which may explain the high proportion of patients on post-treatment PPI.

The management has been different between our tertiary center and other centers. More dilations were performed in the tertiary center, while there were more surgical myotomies elsewhere. This could be explained by a greater endoscopic experience of tertiary center gastroenterologists. Physicians from other centers may have had a little less experience at this level and referred more often to surgery.

One of the strengths of our study is its 7-year duration and its relatively high number of eligible patients. About half of the patients were treated in tertiary centers and the other half in secondary or community centers in various regions of Quebec.

The demographics of our study correlate well with the population suffering from achalasia [2]. Thus, the external validity of this study seems appropriate. One of the limitations of this study is the relatively high proportion of lost to follow-up,

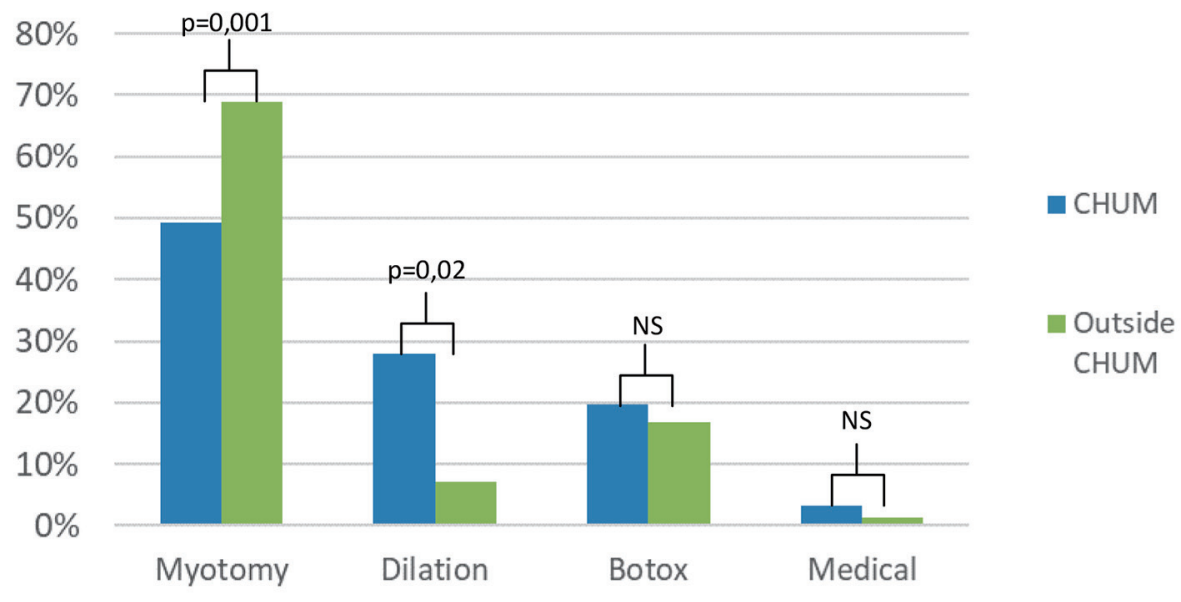

Figure 8. Comparison of the management of patients treated at the CHUM vs. outside CHUM for esophageal achalasia. 


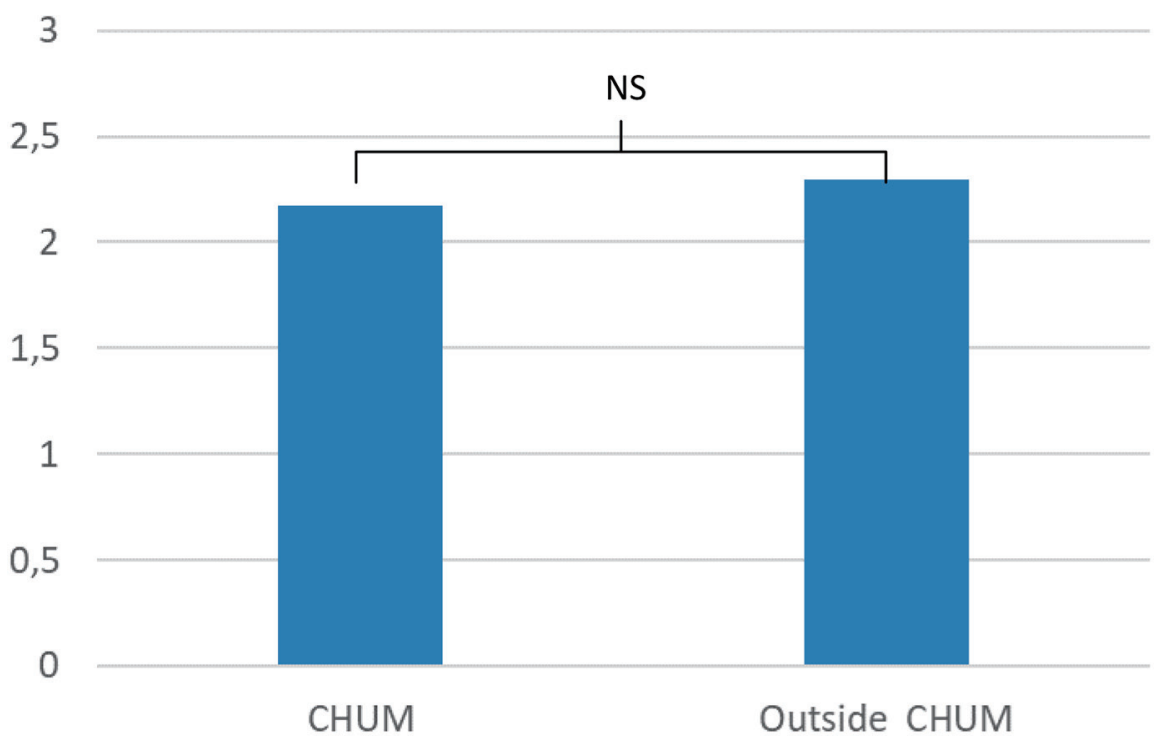

Figure 9. Comparison of post-treatment Eckardt score depending on the site of treatment for esophageal achalasia.

which can create a selection bias.

In conclusion, Heller myotomy remains the most frequent treatment, especially outside our tertiary center. The rate of post-treatment dysphagia remains low, regardless of the type of treatment or the site of management. The need for a second treatment is higher after dilatation and Botox than after Heller myotomy. PPI intake is high regardless of the type and site of treatment, although its relevance has not been evaluated. treatments and we do not know the type of treatments offered in Quebec and their efficacy.

In our study, we found that Heller myotomy stays the most frequent treatment, especially outside the CHUM, a tertiary center.

We did not find a significant difference for the post-treatment Eckardt score depending on the type of treatment, but the need for second treatment was higher after Botox and endoscopic dilation than Heller myotomy.

\section{Key messages}

\section{Acknowledgments}

Esophageal achalasia can be managed with many different We thank Dr Bouin for his help and his comments, and Mrs.

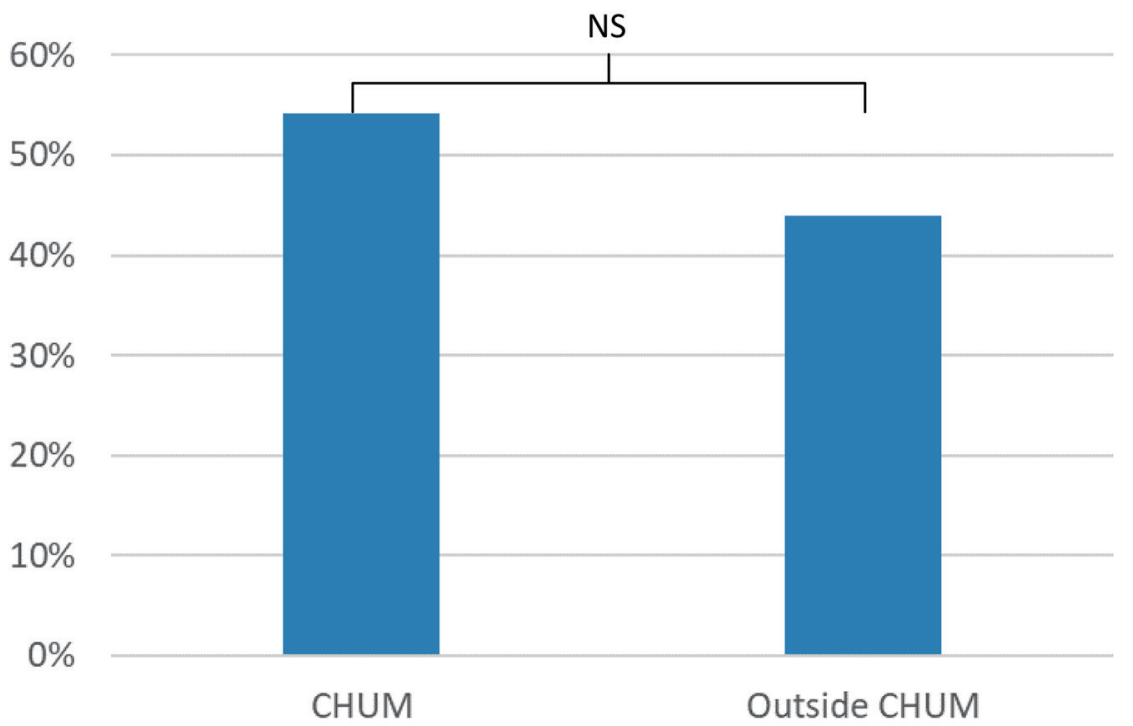

Figure 10. Percentage of patients taking proton pump inhibitor depending on the site of treatment for esophageal achalasia. 
Neshkova for the access to files.

\section{Financial Disclosure}

This study did not receive any funding.

\section{Conflict of Interest}

None to declare.

\section{Informed Consent}

Informed consent was obtained before we included patients in this study.

\section{Author Contributions}

CP performed data collection and analyzed data; EN, DvR and MB designed research study; $\mathrm{CP}$ and $\mathrm{MB}$ drafted manuscript; MB supervised the study.

\section{Abbreviations}

LES: lower esophageal sphincter; POEM: peroral endoscopic myotomy; PPI: proton pump inhibitor; GERD: gastroesophageal reflux disease

\section{References}

1. Patel DA, Lappas BM, Vaezi MF. An overview of achalasia and its subtypes. Gastroenterol Hepatol (N Y). 2017;13(7):411-421.

2. Stavropoulos SN, Friedel D, Modayil R, Parkman HP. Diagnosis and management of esophageal achalasia. BMJ. 2016;354:i2785.

3. Pandolfino JE, Gawron AJ. Achalasia: a systematic review. JAMA. 2015;313(18):1841-1852.

4. Moonen A, Boeckxstaens G. Current diagnosis and man- agement of achalasia. J Clin Gastroenterol. 2014;48(6):484490.

5. Zaninotto G, Bennett C, Boeckxstaens G, Costantini M, Ferguson MK, Pandolfino JE, Patti MG, et al. The 2018 ISDE achalasia guidelines. Dis Esophagus. 2018;31(9):doy071.

6. Jung HE, Lee JS, Lee TH, Kim JN, Hong SJ, Kim JO, Kim HG, et al. Long-term outcomes of balloon dilation versus botulinum toxin injection in patients with primary achalasia. Korean J Intern Med. 2014;29(6):738-745.

7. Wang L, Li YM, Li L. Meta-analysis of randomized and controlled treatment trials for achalasia. Dig Dis Sci. 2009;54(11):2303-2311.

8. Cheng JW, Li Y, Xing WQ, Lv HW, Wang HR. Laparoscopic Heller myotomy is not superior to pneumatic dilation in the management of primary achalasia: Conclusions of a systematic review and meta-analysis of randomized controlled trials. Medicine (Baltimore). 2017;96(7):e5525.

9. Weber CE, Davis CS, Kramer HJ, Gibbs JT, Robles L, Fisichella PM. Medium and long-term outcomes after pneumatic dilation or laparoscopic Heller myotomy for achalasia: a meta-analysis. Surg Laparosc Endosc Percutan Tech. 2012;22(4):289-296.

10. Marano L, Pallabazzer G, Solito B, Santi S, Pigazzi A, De Luca R, Biondo FG, et al. Surgery or peroral esophageal myotomy for achalasia: a systematic review and metaanalysis. Medicine (Baltimore). 2016;95(10):e3001.

11. Talukdar R, Inoue H, Nageshwar Reddy D. Efficacy of peroral endoscopic myotomy (POEM) in the treatment of achalasia: a systematic review and meta-analysis. Surg Endosc. 2015;29(11):3030-3046.

12. Moonen A, Annese V, Belmans A, Bredenoord AJ, Bruley des Varannes S, Costantini M, Dousset B, et al. Long-term results of the European achalasia trial: a multicentre randomised controlled trial comparing pneumatic dilation versus laparoscopic Heller myotomy. Gut. 2016;65(5):732-739.

13. Novais PA, Lemme EM. 24-h pH monitoring patterns and clinical response after achalasia treatment with pneumatic dilation or laparoscopic Heller myotomy. Aliment Pharmacol Ther. 2010;32(10):1257-1265.

14. Ates F, Vaezi MF. The Pathogenesis and management of achalasia: current status and future directions. Gut Liver. 2015;9(4):449-463. 